\title{
Tuning of photonic crystal cavities by controlled removal of locally infiltrated water
}

\author{
Francesca Intonti, ${ }^{1,2, a)}$ Silvia Vignolini, ${ }^{3}$ Francesco Riboli, ${ }^{3}$ Margherita Zani, ${ }^{3}$ \\ Diederik S. Wiersma, ${ }^{3}$ Laurent Balet ${ }^{4}{ }^{4}$ Lianhe H. Li, ${ }^{4, b)}$ Marco Francardi, ${ }^{5}$ \\ Annamaria Gerardino, ${ }^{5}$ Andrea Fiore, ${ }^{6}$ and Massimo Gurioli ${ }^{1,2}$ \\ ${ }^{1}$ CNISM, Unità di Ricerca di Firenze, Via Sansone 1, 50019 Sesto Fiorentino, Italy \\ ${ }^{2}$ Department of Physics and European Laboratory for Non-linear Spectroscopy, University of Florence, \\ Via Nello Carrara 1, 50019 Sesto Fiorentino, Italy \\ ${ }^{3}$ European Laboratory for Non-linear Spectroscopy and INFM-BEC, Via Nello Carrara 1, \\ 50019 Sesto Fiorentino, Italy \\ ${ }^{4}$ Institute of Photonics and Quantum Electronics, Ecole Polytechnique Fédérale de Lausanne, Station 3, \\ CH-1015 Lausanne, Switzerland \\ ${ }^{5}$ Institute of Photonics and Nanotechnology, CNR, via del Cineto Romano 42, 00156 Roma, Italy \\ ${ }^{6}$ COBRA Research Institute, Eindhoven University of Technology, 5600 MB Eindhoven, The Netherlands
}

(Received 11 August 2009; accepted 20 September 2009; published online 29 October 2009)

We present a spectral tuning mechanism of photonic crystal microcavities based on microfluidics. The microinfiltration with water of one or few cavity holes and its subsequent controlled evaporation allow us to tune the cavity resonances in a spectral range larger than $20 \mathrm{~nm}$, with subnanometer accuracy, and we also observe that the addition of water in the microcavity region improves its quality factor Q. (C) 2009 American Institute of Physics. [doi:10.1063/1.3247894]

By merging microfluidics with photonics, it is possible to achieve very functional and compact devices making them tunable, reconfigurable, and flexible. ${ }^{1}$ Microfluidics represents a promising postgrowth approach to face one of the crucial issues in the progress of photonic crystal (PC) microcavities, i.e., the possibility to control the cavity resonances. The combination of PC components with microfluidics has already lead to interesting results like light emitting Si-based devices, ${ }^{2}$ microcavities with high quality factor, ${ }^{3}$ and continuously tunable distributed feedback dye laser. ${ }^{4}$ In this letter, we report on a water microinfiltration at the single pore level of a microcavity on a two-dimensional (2D) PC membrane. Differently from other local and reversible tuning methods, ${ }^{5,6}$ the addition of water modifies the dielectric environment of the microcavity producing a spectral redshift of the resonances larger than $20 \mathrm{~nm}$, which is stable for weeks. In addition, the liquid infiltration improves the optical quality of the microcavity. Furthermore, the controlled evaporation of the infiltrated water allows a local and continuous fine tuning of the resonances, with a precision better than $0.05 \mathrm{~nm}$.

The samples under consideration are 2D PC microcavities on a suspended membrane incorporating quantum dots (QDs) acting as local light sources. They consist of a GaAs based heterostructure: three layers of high-density InAs QDs emitting at $1300 \mathrm{~nm}$ are grown by molecular beam epitaxy at the center of a 320-nm-thick GaAs membrane. ${ }^{7}$ The studied PC structures consist of a two-dimensional triangular lattice of air holes with filling fraction $f=35 \%$, where the cavity is formed by a hole, larger than the pores of the PC, which replaces a central hole and its six nearest neighbors. We investigated samples with different lattice constant $a$, ranging from of 301 to $321 \mathrm{~nm}$, and different diameter of the central

\footnotetext{
a)Electronic mail: intonti@lens.unifi.it.

${ }^{b)}$ Present address: School of Electronic and Electrical Engineering, The University of Leeds, Woodhouse Lane, Leeds LS2 9JT, United Kingdom.
}

hole $d$, ranging from 500 to $650 \mathrm{~nm}$. The inset (I) of Fig. 1 shows a typical topographic image, collected with the probe of a scanning near-field microscope, of the cavity with $a$ $=311 \mathrm{~nm}$ and $d=550 \mathrm{~nm}$. Photoluminescence (PL) spectra of the samples were collected in a confocal configuration using a $50 \times$ microscope objective $(\mathrm{NA}=0.7)$. The sample is excited with light from a diode laser $(\lambda=780 \mathrm{~nm})$ and the emitted PL signal is coupled to a single-mode optical fiber of $6 \mu \mathrm{m}$ core diameter, acting as a confocal pinhole, connected to a spectrometer. The PL signal, dispersed by the spectrometer, is finally collected by a liquid nitrogen cooled InGaAs array. The sample is mounted on an $X Y$ stage so that its lateral position can be scanned in respect to the objective allowing the collection of two-dimensional PL maps. The spatial and spectral resolutions of the experimental setup are $1 \mu \mathrm{m}$ and $1 \mathrm{~nm}$, respectively. A local infiltration apparatus for transferring subfemtoliter amounts of liquids inside the pores ${ }^{8}$ permits to perform a controlled liquid deposition inside the central hole of the cavities.

In the spectral region covered by the QD emission, the PC microcavities exhibit two or three main resonances, as shown in Fig. 1(a), where the PL spectrum of the microcav-

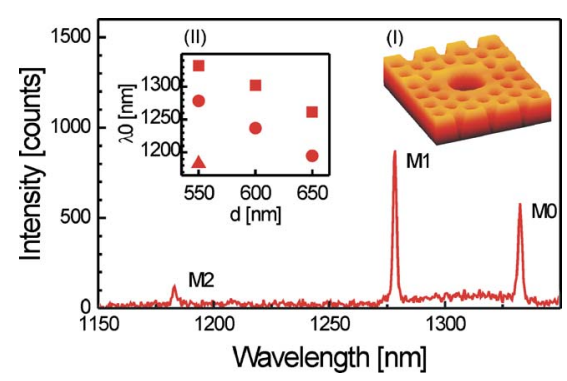

FIG. 1. (Color online) (a) PL spectrum of the cavity with $a=311 \mathrm{~nm}$ and $d=550 \mathrm{~nm}$. Inset (i) topographical image of the sample reporting a $2 \times 2 \mu \mathrm{m}$ region around the cavity. Inset (ii) evolution of the spectral position of the cavity modes vs the diameter of the central hole. Squares correspond to M0, circles to M1, and triangles to M2. 

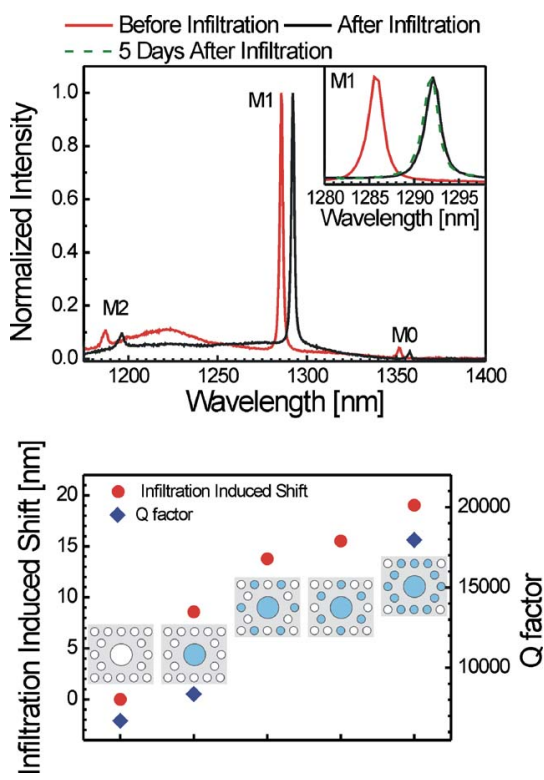

FIG. 2. (Color online) (a) Normalized PL spectra of the cavity with $a=321 \mathrm{~nm}$ and $d=600 \mathrm{~nm}$ before (red line) and after the infiltration process (black line). Inset, zoom on the M1 mode, PL spectra before (red solid line), after the infiltration process (black solid line) and five days after the infiltration process (dashed green line). (b) Calculated infiltration induced spectral shift, red circles, and modification of the $Q$ factor, blue diamonds, for different realization of the infiltration, the gray (blue) pores indicate the ones filled with water $n=1.3$.

ity with $a=311 \mathrm{~nm}$ and $d=550 \mathrm{~nm}$ is reported. Starting from the mode at longer wavelength centered at $1332.5 \mathrm{~nm}$, M0, and moving to shorter wavelength the M1 and M2 modes appear at 1278.5 and $1183.0 \mathrm{~nm}$, respectively. The spectral position of the resonances is highly sensitive to the diameter of the central hole. Inset (II) of Fig. 1 shows the peak position, $\lambda_{0}$, of three cavities with the same lattice constant, $a=311 \mathrm{~nm}$, and different diameters of the central hole, ranging from 550 to $650 \mathrm{~nm}$ in $50 \mathrm{~nm}$ step. The increase of the central hole diameter of the microcavity implies a blue shift of the modes; a $50 \mathrm{~nm}$ increase of the central hole diameter affects the resonances with a blue shift between 31 and $42 \mathrm{~nm}$. Similarly, fixing the diameter of the central hole and decreasing the lattice parameter in $10 \mathrm{~nm}$ steps, produces a blue shift of the resonances of about $50 \mathrm{~nm}$ per step. The behavior of the cavity resonances is well reproduced by finite difference time domain (FDTD) calculations.

We tuned the cavity resonances by locally increasing the average dielectric constant through a controlled microinfiltration of water $(n=1.3)$ in the central hole of the cavities. A typical result of the selective infiltration is shown in Fig. 2(a) for the cavity with $a=321 \mathrm{~nm}$ and $d=600 \mathrm{~nm}$, where the red and black PL spectra were collected before and after the water infiltration, respectively. In this case, the deposition of a volume of water of about $10^{-2}$ femtoliter produces a shift of 6.3, 6.9, and $9.7 \mathrm{~nm}$ for the mode M0, M1, and M2, respectively, and the $Q$ factor does not show any variation. It is interesting to note that this intentional modification of the dielectric environment is stable in time. This can be seen in the inset of Fig. 2(a), where a zoom-in of the M1 mode is shown. The PL spectrum collected five days after the infiltration was performed (dashed green line) is not significantly shifted compared to the PL spectrum collected just after the microinfiltration process (solid black line). ${ }^{8}$ We performed the microinfiltration on several cavities. The optical inspec- tion during the infiltration process shows slightly different infiltration extensions and we measured a spectral shift of the modes between 6.3 and $24.4 \mathrm{~nm}$ depending on the accuracy of the infiltration. We also observed an improvement of the $Q$ factor up to $38 \%$ that, in general, is higher for larger spectral shifts. To quantitatively confirm that the different shifts of the resonances are all due to the local filling of the cavities, we carried out FDTD calculations that simulate the microcavity as grown and with different realization of microinfiltration. The numerical calculations were performed with a commercial three-dimensional FDTD code. Computational meshes were $25 \mathrm{~nm}$, with perfect matching layer of $1.5 \mu \mathrm{m}$. Figure 2(b) shows the results on the spectral position of the M1 mode (red circles). We calculated a spectral shift of 8.6 $\mathrm{nm}$ when the central hole is filled with water, while filling also the 12 pores surrounding the cavity produces a resonance shift as large as $19.0 \mathrm{~nm}$. The two intermediate situations, where alternatively the first or second neighbor pores around the microcavity are infiltrated, indicate that the infiltration of the first neighbor pores has a larger influence on the spectral position of the resonance. Comparing these calculations and the experimental results of Fig. 2(a) we conclude that in the case of the cavity with $a=321 \mathrm{~nm}$ and $d$ $=600 \mathrm{~nm}$ we were indeed able to target only the central hole with the infiltration.

Figure 2(b) shows the calculated quality factor of the M1 mode (blue diamonds). When only the central hole is filled there is a slight improvement of $Q$, while filling also the twelve pores surrounding the cavity the $Q$ factor doubles. This latter consistent increase of $Q$ is likely due to the combined result of three different effects. One is the fact that the filling of the pores around the cavity implies a softer confinement of the light due to a decrease of the refractive index contrast. ${ }^{9}$ The second effect is that in general the larger the cavity the larger the $Q$ is, and the third one is that the increase of the average refractive index, due to the water infiltration, may reduce the out-of-plane losses. The FDTD calculations confirm the overall picture, even if the experimental measured $Q$ and its observed increase, due to water infiltration, are lower than the predicted ones. This discrepancy is probably due to fabrication imperfections, residual absorption by both the QDs and the infiltrated water, and non perfectly symmetric realization of the water infiltration.

At room temperature the infiltrated water remains in the pores for several days, but if the sample is heated the water evaporation could be speeded up and the control of the evaporation rate could be exploited for fine tuning the cavity resonances. We were able to locally heat the sample by using our PL setup at high excitation density. The dissipation of the absorbed excitation power causes an increase of the cavity temperature that in turn raises the value of the dielectric constant. This modification of the dielectric constant is observed by a red shift of the cavity resonances. ${ }^{10,11}$ Figure 3(a) reports, on a blue to white scale, the two-dimensional PL map associated with the M1 mode of the cavity with $a=311 \mathrm{~nm}$ and $d=600 \mathrm{~nm}$ obtained with a low excitation density $(2.5$ $\times 10^{5} \mathrm{~W} / \mathrm{cm}^{2}$ ). The signal appears as a circular spot that stems at the microcavity position with a lateral extension of $1 \mu \mathrm{m}$, which defines the spatial resolution of our setup. By increasing the excitation density $\left(1.3 \times 10^{6} \mathrm{~W} / \mathrm{cm}^{2}\right)$ we observed that during the scan, when the excitation approaches 
(a)

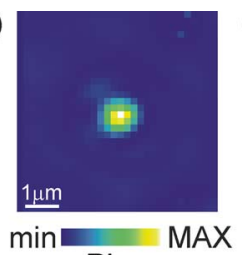

(b)
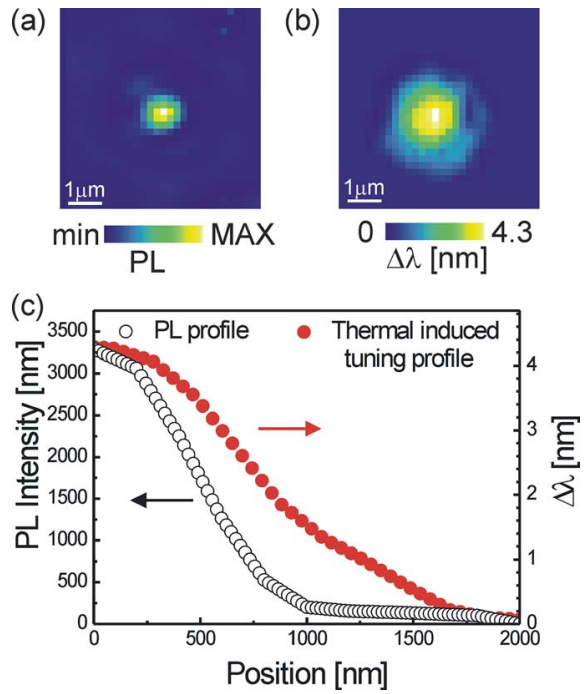

FIG. 3. (Color online) (a) Spatial distribution of the PL signal associated with the M1 mode for the cavity with $a=311 \mathrm{~nm}$ and $d=600 \mathrm{~nm}$ collected with low excitation density. (b) Map of the temperature gradient obtained with high excitation density. Both images cover an area of $6 \times 6 \mu \mathrm{m}$. (c) Radial averaged profiles of the PL map (open circles) and of the temperature gradient map (closed circles).

the center of the cavity, the M1 resonance shifts to the red more than $4 \mathrm{~nm}$ with respect to the case when the laser is focused outside the cavity, indicating that the microcavity is locally heated. Therefore, in the assumption that the temperature gradient is independent of the spatial position on the cavity and that its extension is larger than the one of the cavity mode, the spectral position of the cavity resonance gives an indication on the local temperature. ${ }^{11}$ By extracting at every position of the scan the spectral position of the cavity we reconstructed the map of the thermal induced tuning shown on a blue to white scale in Fig. 3(b). Figure 3(c) compares the radial averaged cross sections of Figs. 3(a) and 3(b). Since at a distance smaller than $2 \mu \mathrm{m}$ the temperature induced tuning is already negligible, the excitation laser heats indeed locally. To have a crude estimation of the cavity temperature we consider a thermal shift of the resonance of $0.12 \mathrm{~nm} / \mathrm{K},{ }^{11}$ that results in maximum temperature increase of $36 \mathrm{~K}$.

We made use of this local rise in temperature to evaporate gradually the locally infiltrated water. For this purpose we focused our attention on a second infiltration experiment, that is the infiltration of the cavity with $a=311 \mathrm{~nm}$ and $d=600 \mathrm{~nm}$. The time evolution of the M1 mode spectral position, as a consequence of the water infiltration and of the successive heating, is shown in Fig. 4. After the infiltration we observed a shift of the modes of about $15 \mathrm{~nm}$ and an improvement of the $Q$ factor of $37 \%$, indicating that apart the central hole we inserted water in a couple of pores around the cavity [Fig. 2(b)]. We then heated up the sample by increasing the laser intensity and, at different time intervals, we decreased the excitation intensity to check the position of the resonances. The data related to the M1 mode are reported in Fig. 4. After 100 min the mode shows already a blue shift

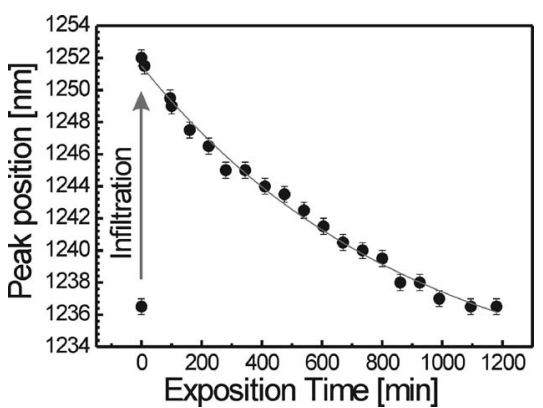

FIG. 4. Time evolution of the spectral position of the M1 modes of the cavity with $a=311 \mathrm{~nm}$ and $d=600 \mathrm{~nm}$. At time zero we performed the infiltration and after we heated the cavity. The solid line is a guideline to the eye.

of $2.5 \mathrm{~nm}$. To completely empty the microcavity the sample was heated for almost $20 \mathrm{~h}$. The slow blue shift rate, of the order of $10^{-2} \mathrm{~nm} / \mathrm{min}$, allows a fine continuous tuning of the microcavity.

In conclusion, the manipulation of water volumes of the order of few atto liters inside a PC microcavity permits a fine continuous tuning over several nanometers of the resonances of PC microcavities and, unlike other tuning strategies, an increase of the $Q$ factor of the microcavity. Finally, since we observed that the filling of the first neighbor pores has a large influence both on the resonance spectral position and on the $Q$ factor, this technique can also be used to post-growth control PC microcavities with any kind of geometry.

The author thanks Lucio Claudio Andreani for important contributions during the initial stage of this work. L.B and A.F. acknowledge financial support from the Swiss National Science Foundation n. PP0022-112405.

${ }^{1}$ C. Monat, P. Domachuk, and B. J. Eggleton, Nat. Photonics 1, 106 (2007). ${ }^{2}$ S. Vignolini, F. Riboli, F. Intonti, M. Belotti, M. Gurioli, Y. Chen, M. Colocci, L. C. Andreani, and D. S. Wiersma, Phys. Rev. E 78, 045603 (2008).

${ }^{3}$ U. Bog, C. L. C. Smith, M. W. Lee, S. Tomljenovic-Hanic, C. Grillet, C. Monat, L. O'Faolain, C. Karnutsch, T. F. Krauss, R. C. McPhedran, and B. J. Eggleton, Opt. Lett. 33, 2206 (2008).

${ }^{4}$ Z. Li, Z. Zhang, A. Scherer, and D. Psaltis, Opt. Express 14, 10494 (2006).

${ }^{5}$ F. Intonti, S. Vignolini, F. Riboli, A. Vinattieri, D. S. Wiersma, M. Colocci, L. Balet, C. Monat, C. Zinoni, L. H. Li, R. Houdré, M. Francardi, A. Gerardino, A. Fiore, and M. Gurioli, Phys. Rev. B 78, 041401(R) (2008).

${ }^{6}$ A. Faraon, D. Englund, I. Fushman, J. Vučković, N. Stolz, and P. Petroff, Appl. Phys. Lett. 90, 213110 (2007).

${ }^{7}$ M. Francardi, L. Balet, A. Gerardino, C. Monat, C. Zinoni, L. H. Li, B. Alloing, N. Le Thomas, R. Houdré, and A. Fiore, Phys. Status Solidi C 3, 3693 (2006).

${ }^{8}$ F. Intonti, S. Vignolini, V. Turck, M. Colocci, P. Bettotti, L. Pavesi, S. L. Schweizer, R. Wehrspohn, and D. S. Wiersma, Appl. Phys. Lett. 89, 211117 (2006); Italian Patent No. TO2006A000216 (2006), Extension Europe, USA 27/09/2007, No. WO2007/107959 A1 (2007).

${ }^{9}$ Y. Akahane, T. Asano, B. Song, and S. Noda, Nature (London) 425, 944 (2003).

${ }^{10}$ I. Fushman, E. Waks, D. Englund, N. Stoltz, P. Petroff, and J. Vučković, Appl. Phys. Lett. 90, 091118 (2007).

${ }^{11}$ S. Vignolini, F. Intonti, L. Balet, M. Zani, F. Riboli, A. Vinattieri, D. S. Wiersma, M. Colocci, L. Li, M. Francardi, A. Gerardino, A. Fiore, and M. Gurioli, Appl. Phys. Lett. 93, 023124 (2008). 